\title{
The role of the multidisciplinary emphysema team meeting in the provision of lung volume reduction
}

\author{
Inger Oey ${ }^{1}$, David Waller ${ }^{2}$ \\ ${ }^{1}$ Department of Thoracic Surgery, Glenfield Hospital, Leicester, UK; ${ }^{2}$ Department of Thoracic Surgery, St. Bartholomew's Hospital, London, UK \\ Contributions: (I) Conception and design: D Waller; (II) Administrative support: All authors; (III) Provision of study materials or patients: All authors; \\ (IV) Collection and assembly of data: I Oey; (V) Data analysis and interpretation: All authors; (VI) Manuscript writing: All authors; (VII) Final \\ approval of manuscript: All authors. \\ Correspondence to: David Waller. Department of Thoracic Surgery, St. Bartholomew's Hospital, West Smithfield, London, EC1A 7BE, UK. \\ Email: david.waller1@nhs.net.
}

\begin{abstract}
Despite a positive result in favour of lung volume reduction surgery (LVRS), from one of the largest randomized controlled trial in thoracic surgery, the identification of poor outcome in certain high-risk groups has resulted in a worldwide decrease in its utilization. Patient selection is the key to successful lung volume reduction which, with the advent of a range of less invasive techniques, has become more complex. The greater variety of potential therapeutic options will inevitably lead to debate amongst treating clinicians. Therefore, to be able to make an informed decision on the best treatment for an individual patient, discussion between clinicians in a multidisciplinary team (MDT) meeting is advisable. The membership of this MDT must include all specialists involved in assessment and subsequent treatment of the patient including nonmedical input. There must be robust administrative organization and record of decisions together with interdisciplinary communication of decisions. Whilst ultimately it is the patient who will benefit from the MDT, individual participants will enhance their continued professional development. The referral pathway into the MDT must be clearly defined and disseminated. Which investigations are to be performed by referrers and which by the specialist centre need to be in an agreed protocol. Specialist input may be required to interpret the results of the latest assessment tools. The decision-making process of the MDT begins with confirmation of basic selection criteria but addresses three main areas of discussion: the definition of target areas of lung for reduction; the presence of collateral, interlobar ventilation and an assessment of individualized risk and benefit. The emphysema or lung volume reduction MDT has been established in several specialist units and its benefits include an increase in referrals overall for LVR. The establishment of an MDT approach to lung volume reduction has now been incorporated into several national guidelines.
\end{abstract}

Keywords: Multidisciplinary team (MDT); emphysema; lung volume reduction

Submitted Dec 04, 2017. Accepted for publication Feb 06, 2018.

doi: $10.21037 /$ jtd.2018.02.68

View this article at: http://dx.doi.org/10.21037/jtd.2018.02.68

\section{Introduction}

Despite a positive result for lung volume reduction surgery (LVRS) in the NETT trial (1), the uptake of surgical LVR has been limited due to a perception of high mortality attached to surgery (2).

There has been considerable evolution in the technique of lung volume reduction since the report by Cooper $e t a l$. of a bilateral procedure through a median sternotomy (3). In an attempt to reduce complications and to extend the benefits of LVR some surgeons adopted a staged bilateral video-assisted thoracoscopic (VAT) approach to LVR $(4,5)$. In addition pulmonologists have developed potentially less invasive bronchoscopic techniques. Some are in experimental stages, others have become more established $(6,7)$. As a result of all these developments there is now a 
range of techniques available for patients with emphysema, which can be confusing for the patient population as well as health professionals.

Patient selection is the key to successful lung volume reduction and with the advent of more techniques has become more complex. Accurate assessment of the lung targets for LVR may need a consensus opinion. Endobronchial valves (EBV) are only considered in patients without collateral ventilation $(\mathrm{CV})$ which needs to be excluded through further investigation (8). Most importantly, as LVR is primarily a procedure to improve health status, there is a need for an accurate risk-benefit analysis to assess which patients should proceed to which method of LVR.

The complexity of assessment and variety of treatments will inevitably result in a difference of opinion between health professionals, and patients, hence a meaningful discussion at a multidisciplinary team meeting (MDT) is mandatory.

\section{The LVR MDT}

\section{Membership}

It is advisable to have at least two thoracic surgeons as core members of the MDT to cover absences and to form a consensus in borderline cases. In our experience both surgeons should be experienced in video-assisted thoracoscopic surgery (VATS) and endobronchial techniques to avoid bias and maintain equipoise. This does not preclude interventional pulmonologists forming an integral part of the team. Other physicians who specialize in chronic obstructive pulmonary disease (COPD) and who may attract tertiary complex referrals are a mainstay. Ideally, they will have expertise in respiratory physiological measurement.

Whilst the presence of a member of the lung transplant team is desirable at the LVR MDT it is not mandatory provided that this treatment option is considered in appropriate cases. Certainly, LVR MDTs and the clinical programs should not be limited to centres that can also provide lung transplantation.

Detailed analysis of imaging will require radiologists with an understanding of thoracic CT and the latest software for emphysema analysis and training in nuclear medicine to interpret isotope images.

Preoperative pulmonary rehabilitation is a requirement for lung volume reduction as it conditions patients for
LVR and also helps with informed consent. Therefore, physiotherapy input is important.

The administrator is responsible for streamlining the referrals to the MDT, which may be from physicians as well as from surgeons, to take minutes and to distribute these after the meeting. A clear referral proforma (Figure 1) will help to ensure relevant investigations have been performed before the MDT discussion, streamlines the discussion at the meeting, and will help with data collection.

\section{Evidence of MDT value}

In our own centre we correlated the advent of the LVR MDT with a steady increase in referrals for all forms of LVR and the proportion of referred patients who were accepted for LVR (9). Education of referring respiratory physicians and dissemination of feedback may also have helped in improving the referrals of suitable patients. In the last 10 years we saw an increase in acceptance rate from one third to one half of all referred patients.

In most cases, over $25 \%$ of patients, the reason for unsuitability was the absence of target areas. Another quarter of patients were considered too good, either by the medical team or because the patient felt too good and therefore did not feel the need for LVR. Patients in respiratory failure included those with hypercapnia and/or pulmonary hypertension.

We also found that 16 patients who were initially felt not to be suitable where subsequently re-discussed and offered surgery. The median time between initial MDT discussion and surgery (after subsequent MDT decision) was 19 months (range 11 months to 6 years). The reasons patients initially did not proceed to LVR and subsequently underwent LVR were diffuse disease without targets which changed subsequently, deterioration in patient's functional status and patient's choice.

\section{The patient pathway-what is the route into the MDT?}

The initial referral for LVR from non-specialist centres is ideally seen in a dedicated outpatient clinic for advanced emphysema, which is jointly staffed by the respiratory medicine and thoracic surgery departments. Prior to referral patients should have been medically optimised and have completed pulmonary rehabilitation. In general patients will have had lung function testing but it is important to confirm that static lung volumes were tested 
Glenfield Hospital, Leicester: Lung Volume Reduction MDT

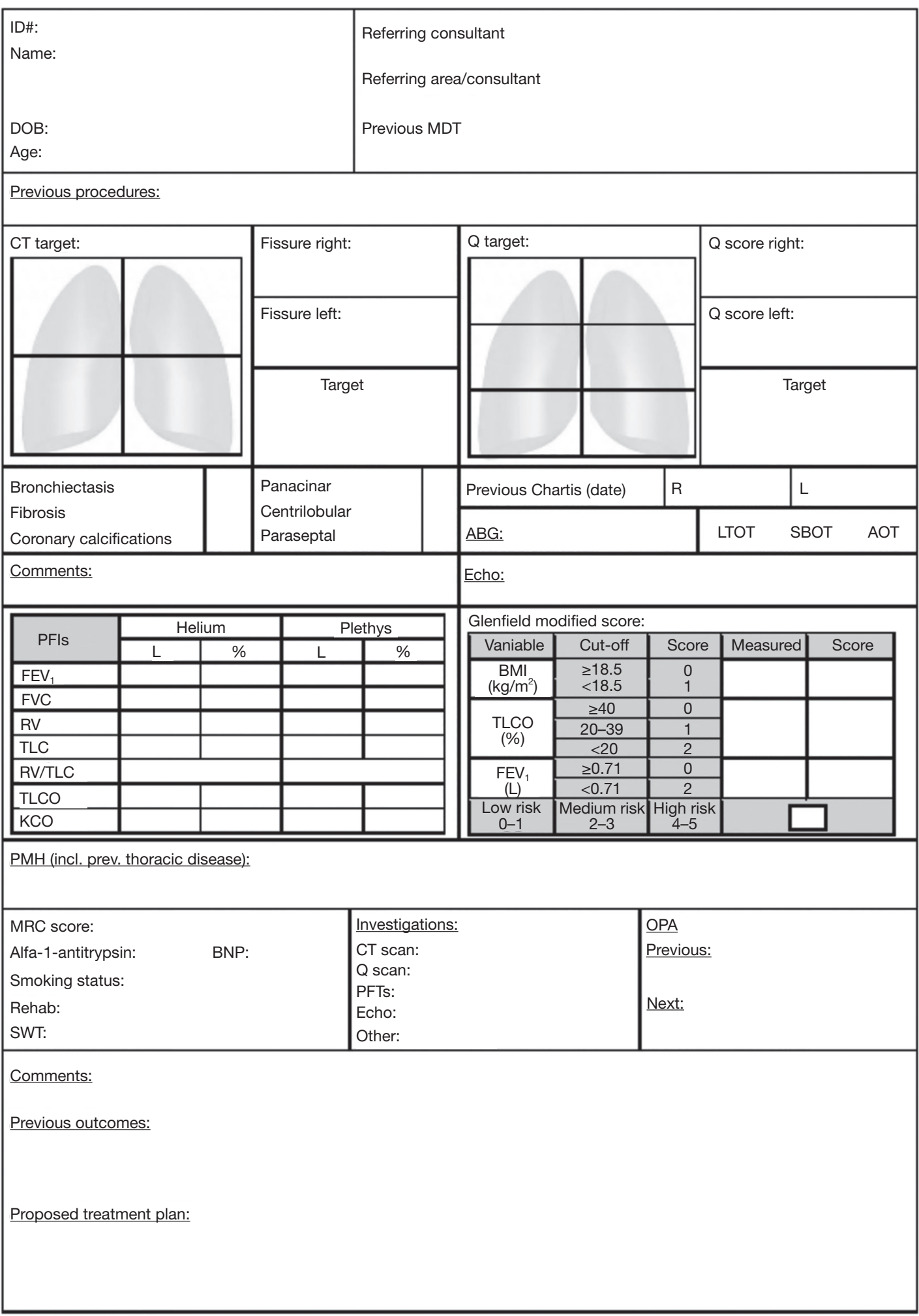

Figure 1 Referral proforma for use in LVR MDT. LVR, lung volume reduction; MDT, multidisciplinary team. 
by body box plethysmography and not by helium dilution (which may underestimate) and if necessary these may need to be repeated prior to the initial consultation. All patients need a recent high-resolution (HR) CT scan and if possible echocardiography.

It has been our practice, to avoid inter-technique variability, for the referred patients to undergo quantitative radionuclide lung scintigraphy in our centre in order to further determine the target areas for volume reduction. Similarly, identification of likely CV by either CT software or Chartis bronchoscopic catheter (Chartis system ${ }^{\circledR}$, Pulmonx, Redwood City, CA, USA) is centralised but may follow on from the initial consultation.

It is advisable for all patients discussed at the MDT to have been seen previously in person by at least one member of the team.

\section{MDT selection process}

During the MDT meeting patient's suitability is discussed with a completed proforma and the relevant radiology investigations visible to all. Teleconferencing may facilitate wider participation.

Initially, it is established that the case meets the accepted basic inclusion criteria. As a guideline we accept patients with severe airway obstruction [forced expiratory volume $\left(\mathrm{FEV}_{1}\right)$ between $20 \%$ pred and $40 \%$ pred) and with hyperinflation (TLC over $120 \%$ pred and RV over $200 \%$ pred, with RV: TLC $>55 \%$ ) and who have a relatively preserved transfer factor (over $20 \%$ pred). Although the NETT trial (1) excluded patients with hypercapnia there is some evidence that in selected patients' lung volume reduction can benefit these patients (10) and this should probably be viewed as a relative exclusion criterion.

In potentially suitable patients we then look at three aspects: the presence and location of target areas, the presence or absence of $\mathrm{CV}$ and the individualised risk score.

It is accepted that LVR in patients with heterogeneous emphysema has better postoperative results than in those with homogeneous emphysema (11). We advocate correlating the images obtained by CT and perfusion scintigraphy. Obviously when a lung perfusion scan does show reduced perfusion, it will need to be confirmed by CT scan that this is due to the presence of emphysema. Similarly, a lung perfusion scan is a functional scan and we have seen examples where there is discordance with the anatomical CT scan.

A CT scan is important to not only confirm the presence of emphysema but also to exclude other conditions i.e. lung cancer, bronchiectasis. In some cases, it may determine the surgical strategy for lung volume reduction, e.g., the present of a lung cancer can be combined with VAT LVRS $(12,13)$.

A CT scan can also help to predict the likely presence or absence of CV. Rather than the traditional cross-sectional views other views may help to look for fissure integrity and novel CT software analysis is now available that quantifies emphysema and which can also look for fissure integrity and so improve the success rate of $\operatorname{EBV}(8,14)$. We do advocate subsequent bronchoscopy and direct measurement of $\mathrm{CV}$ using the Chartis system ${ }^{\circledR}$ to further consolidate the decision making (15).

Many patients have risk factors for cardiovascular disease and echocardiography is essential in assessment. In the presence of emphysema pulmonary hypertension needs to be excluded. It can be difficult in emphysematous patients to obtain adequate views on echocardiograms (16). Additional investigations to assess cardiac status are measuring brain natriuretic peptide (BNP) levels, and CT scan can give a coronary calcification score. In some cases, a cardiac catheterisation study may be required.

After excluding patients with the above risk factors, an analysis by Greening et al. found that three further factors could be used to predict the case-specific risk of operative mortality from LVRS: body mass index (BMI), $\mathrm{FEV}_{1}$ (in lit) and transfer factor $\left(\mathrm{D}_{\mathrm{LCO}}\right)(17)$. According to this "BFG risk score" patients can be categorised into three risk groups; low mortality, moderate mortality and high mortality by the MDT in order to inform further management discussion (Figure 2).

Since we have adopted a policy of staged unilateral LVR, patients who have had initial LVR and who want to be considered for a further procedure are referred to the MDT meeting in the same way and undergo similar assessment $(4,5)$.

\section{The following are the most common selection scenarios subject to MDT discussion.}

In patients who are $\mathrm{CV}$ positive (so not suitable for EBV), with good target areas and low BFG risk score, we advocate a surgical VAT procedure. Even in patients with homogeneous emphysema, without clear target areas, we would consider VAT LVRS if they are very hyperinflated (18). In CV negative patients with good target areas, but with moderate risk, we would now propose EBV. CV negative patents with good target areas with low risk one could 


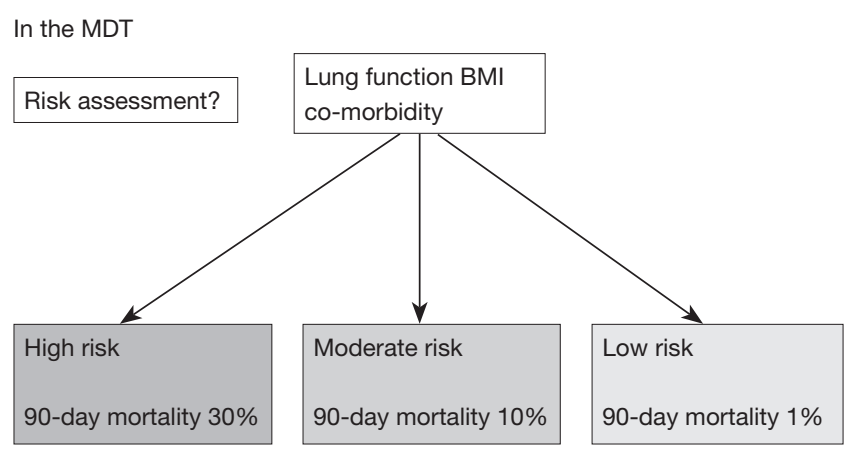

Figure 2 Risk assessment in the LVR MDT (17). BMI, body mass index; LVR, lung volume reduction; MDT, multidisciplinary team.

consider either a VATS or EBV procedure There is currently an ongoing trial in the UK to compare the two (CELEB trial, ISRCTN19684749). Patients with moderate risk who are not suitable for $\mathrm{EBV}$, as they are $\mathrm{CV}$ positive, form the most difficult MDT discussion group. Entry into clinical trials of other endobronchial techniques i.e., coils should be discussed with the patient.

Patients in the higher risk category for LVRS who may have CV may still be eligible for lung transplantation. Previous LVR is not a contraindication to subsequent lung transplant and although it can increase the risk of perioperative complications it may also improve the general condition of the patient and can be considered as bridging procedure (19).

\section{Who benefits from the MDT?}

In our experience each individual member has found benefit from MDT discussions. The respiratory physicians and radiologists receive feedback on outcome related to their preoperative assessment. Particularly in the case of nonspecialists, a change in their perception of risk, following feedback, may result in an increased number of referrals.

By discussing the results of patients, the surgeon will get a better appreciation of respiratory physiology and see how this is applied in surgical physiology. Operative planning will be enhanced by identifying targets and the choice between LVRS and EBV will be informed.

Finally, the patient gains most. Their management is discussed amongst a group of experienced health professionals. When all factors are taken into consideration there may be a change of approach or a major decision about suitability.

\section{Conclusions}

MDT is important to increase referral of suitable patients and to ensure appropriate treatment. It avoids individual preferences i.e., when EBVs are done in isolation. The MDT is important for central data collection for future audit and research. Many of the newer techniques are part of various local and national trials. The MDT will help with entry into these registries.

The MDT should encourage to "cast the net wide", facilitating assessment and avoiding preselection bias by non-specialists. Our MDT referral advice is to send all symptomatic patients seeking further intervention, who are hyperinflated and who are not in respiratory or cardiac failure. The specialist centres can then assess further the target areas and whether CV is present and so which patients are suitable and make a choice of which procedure.

It is it important to ensure feedback as good outcomes encourage further referrals. By keeping the referring physicians up-to-date ensures joint ownership in longer term follow-up and timely re-assessment for the next stage LVR.

Finally, all the organisation and administration require adequate investment in both clinical time and finance.

\section{Acknowledgements}

None.

\section{Footnote}

Conflicts of Interest: The authors have no conflicts of interest to declare.

\section{References}

1. Fishman A, Martinez F, Naunheim K, et al. A randomized trial comparing lung-volume-reduction surgery with medical therapy for severe emphysema. $\mathrm{N}$ Engl J Med 2003;348:2059-73.

2. McNulty W, Jordan S, Hopkinson NS. Attitudes and access to lung volume reduction surgery for COPD: a survey by the British Thoracic Society. BMJ Open Respir Res 2014;1:e000023.

3. Cooper JD, Trulock EP, Triantafillou AN, et al. Bilateral pneumectomy (volume reduction) for chronic obstructive pulmonary disease. J Thorac Cardiovasc Surg 1995;109:106-16; discussion 116-9. 
4. Waller D, Oey I. Staged lung volume reduction surgery-rationale and experience. Thorac Surg Clin 2009;19:187-92.

5. Oey IF, Morgan MD, Spyt TJ, et al. Staged bilateral lung volume reduction surgery - the benefits of a patient-led strategy. Eur J Cardiothorac Surg 2010;37:846-52.

6. Sciurba FC, Ernst A, Herth FJ, et al. A randomized study of endobronchial valves for advanced emphysema. N Engl J Med 2010;363:1233-44.

7. Shah PL, Zoumot Z, Singh S, et al. Endobronchial coils for the treatment of severe emphysema with hyperinflation (RESET): a randomised controlled trial. Lancet Respir Med 2013;1:233-40.

8. Davey C, Zoumot Z, Jordan S, et al. Bronchoscopic lung volume reduction with endobronchial valves for patients with heterogeneous emphysema and intact interlobar fissures (the BeLieVeR-HIFi study): a randomised controlled trial. Lancet 2015;386:1066-73.

9. Rathinam S, Oey I, Steiner M, et al. The role of the emphysema multidisciplinary team in a successful lung volume reduction surgery programme. Eur J Cardiothorac Surg 2014;46:1021-6; discussion 1026.

10. Ariyaratnam P, Tcherveniakov P, Milton R, et al. Is preoperative hypercapnia a justified exclusion criterion for lung volume reduction surgery? Interact Cardiovasc Thorac Surg 2017;24:273-9.

11. Hamacher J, Bloch KE, Stammberger U, et al. Two years' outcome of lung volume reduction surgery in different morphologic emphysema types. Ann Thorac Surg 1999;68:1792-8.

Cite this article as: Oey I, Waller D. The role of the multidisciplinary emphysema team meeting in the provision of lung volume reduction. J Thorac Dis 2018;10(Suppl 23):S2824S2829. doi: 10.21037/jtd.2018.02.68
12. Vaughan P, Oey I, Nakas A, et al. Is there a role for therapeutic lobectomy for emphysema? Eur J Cardiothorac Surg 2007;31: 486-90; discussion 490.

13. Perikleous P, Bilancia R, Oey I, et al. Hybrid bilobectomy for treatment of an early-stage lung cancer in a patient with severe emphysema using the benefits of lung volume reduction. Eur J Cardiothorac Surg 2017;52:1000-2.

14. van Rikxoort EM, Goldin JG, Galperin-Aizenberg M, et al. A method for the automatic quantification of the completeness of pulmonary fissures: evaluation in a database of subjects with severe emphysema. Eur Radiol 2012;22:302-9.

15. Koster TD, van Rikxoort EM, Huebner RH, et al. Predicting lung volume reduction after endobronchial valve therapy is maximized using a combination of diagnostic tools. Respiration 2016;92:150-7.

16. Fisher MR, Criner GJ, Fishman AP, et al. Estimating pulmonary artery pressures by echocardiography in patients with emphysema. Eur Respir J 2007;30:914-21.

17. Greening NJ, Vaughan P, Oey I, et al. Individualised risk in patients undergoing lung volume reduction surgery: the Glenfield BFG score. Eur Respir J 2017;49. pii: 1601766.

18. Weder W, Tutic M, Lardinois D, et al. Persistent benefit from lung volume reduction surgery in patients with homogeneous emphysema. Ann Thorac Surg 2009;87:22936; discussion 236-7.

19. Inci I, Iskender I, Ehrsam J, et al. Previous lung volume reduction surgery does not negatively affect survival after lung transplantation. Eur J Cardiothorac Surg 2018;53:596-602. 